\section{On Uniform Convergence in Some Classes of Functions *).}

By

\section{Nosarzewska (Wroclaw).}

The following theorem is well known ${ }^{1}$ ):

(*) If a sequence of monotonic functions defined in an open interval converges everywhere to a continuous function $f$, then it converges almost uniformly ${ }^{2}$ ) to $f$.

The question arises whether an analogous theorem holds for other classes of functions, i. e. whether, for another class of functions, the pointwise convergence or another weaker kind of convergence always implies almost uniform convergence ${ }^{3}$ ). The classes examined in this paper are: the class of all convex functions, the class of all functions convex relative to a family $\Phi$ of functions, the class of all functions the $p$-th difference of which has a constant sign ${ }^{4}$, and the class of all subharmonic functions on a plane domain

Suitable examples ( $v .5$ ) show that the answer is negative in the case of subharmonic functions.

The answer is affirmative for the remaining classes of functions mentioned above (v. 2-4). Besides the known kinds of con-

*) This paper contains the chief results from the author's doctoral thesis presented at the University in Wroclaw, 1949. The paper was presented at the Polish Mathematical Society, Wrocław Section, on November 19, 1948.

1) See e.g. G. Polya und G. Szegö, Aufgaben und Lehrsälze aus der Analysis, Band I, Berlin 1925, p. 63 and 126. See also E. Marczersski et Mr. No. Barzewska, Sur la convergence uniforme et la mesurabilité relative, Colloquium athematicum 1 (1947), pp. 15-18.

2) The definition of almost uniform convergence is given on p. 40. The word "almost" should be omitted in the case of a closed interval.

This question was posed by E. Marczewski.

H. Steinhaus.
Uniform Convergence in Some Classes

vergence (pointwise convergence, asymptotic convergence, convergence in a dense subset) we shall examine a still weaker notion called the pointwise approximation of a function by a sequence of functions. It will be proved that, for the classes of functions, considered in $2-4$, pointwise approximation always implies almost uniform convergence.

1. Pointwise approximation and various kinds of convergence. The letter $I$ will always denote a fixed open interval $a<x<b$. All functions considered in $1-4$ are real and defined in $I$.

A set $A C I$ is said to be $\eta$-dense $(\eta>0)$ if for every $x \in I$ there is an $x_{0} \in A$ such that $\left|x-x_{0}\right|<\eta$. Clearly every dense subset of $I$ is $\eta$-dense for every $\eta>0$, and every measurable set $Z C I$ with mes $(I-Z)<\eta$ is $\eta$-dense.

A sequence of functions $\left\{f_{n}\right\}$ is said to approximate pointwise a function $f$ provided that, given $\varepsilon>0$ and $\eta>0$, there is an integer $N$ such that the set

$$
\underset{x}{E}\left(\left|f_{n}(x)-f(x)\right|<\varepsilon\right)
$$

is $n$-dense for every $n>N$.

For instance, the sequence $\{\sin n x\}$ approximates pointwise every continuous function $f$ such that $|f(x)| \leqslant 1$. This example shows that a sequence $\left\{f_{n}\right\}$ may pointwise approximate an infinite number of functions $f$. Therefore pointwise approximation cannot be considered as a kind of convergence.

The following simple lemmas show that pointwise approximation is a weaker notion than the kinds of converegnce usually considered.

Lemma 1. If $S$ is a dense subset of $I$ and $\lim f_{n}(x)=f(x)$ for every $x \in S$, then the sequence $\left\{f_{n}\right\}$ approximates pointwise the function $f$.

Let $\varepsilon>0, \eta>0$, and let $S_{0}$ be an $\eta$-dense finite subset of $S$. clearly there is an integer $N$ such that

$$
\left|f_{n}(x)-f(x)\right|<\varepsilon \text { for } n>N \text { and } x \in S_{0} .
$$

The set $\underset{x}{E}\left(\left|f_{n}(x)-f(x)\right|<\varepsilon\right)$ is thus $\eta$-dense for $n>N$. 
Lemma 2. If a sequence $\left\{f_{n}\right\}$ of measurable functions converges asymptotically ${ }^{5}$ ) to a measurable function $f$, then $\left\{f_{n}\right\}$ approximates pointwise the function $f$.

Given $\varepsilon>0$ and $\eta>0$, there is an. $N$ such that

$$
\operatorname{mes}\left(\underset{x}{E}\left(\left|f_{n}(x)-f(x)\right|>\varepsilon\right)\right)<\eta \text { for } n>N \text {. }
$$
q. e. d.

Thus the set $\underset{x}{E}\left(\left|f_{n}(x)-f(x)\right|<\varepsilon\right)$ is $\eta$-ilense for every $n>N$,

A sequence $\left\{f_{n}\right\}$ of functions on $I$ is said to converge almost uniformly ${ }^{6}$ ) to a function $f$ if $\left\{f_{n}\right\}$ converges uniformly to $f$ on every closed interval $I_{0} \subset I$.

Lemma 3. In order that a sequence $\left\{f_{n}\right\}$ converge almost uniformly to a function $f$ and that this limit function $f$ be continuous, it is necessary and sufficient that, for every $x_{0} \in I$ and for every $\varepsilon>0$, there be an $\eta>0$ and an integer $N$ such that

(1) $\quad\left|f_{n}(x)-f\left(x_{0}\right)\right|<\varepsilon$ for $n>N$ and $\left|x-x_{0}\right|<\eta$.

The necessity follows from the inequality

$$
\left|f_{n}(x)-f\left(x_{0}\right)\right| \leqslant\left|f_{n}(x)-f(x)\right|+\left|f(x)-f\left(x_{0}\right)\right| .
$$

To prove the sufficiency we first notice that $f(x)=\lim f_{n}(x)$ for every $x \in I$ on account of (1). The condition (1) implies that

$$
\left|f(x)-f\left(x_{0}\right)\right| \leqslant \varepsilon \text { if }\left|x-x_{0}\right|<\eta
$$

which proves the continuity of $f$.

We infer from (1) and (2) that

$$
\left|f_{n}(x)-f(x)\right|<2 \varepsilon \text { if } n>N \text { and }\left|x-x_{0}\right|<\eta \text {. }
$$

Let $I_{0} \subset I$ be a closed interval. For every $x_{0} \in I_{0}$ there exists a neighbourhood $\left|x-x_{0}\right|<\eta$ where (3) holds. By the Heine-Borel Theorem there is a finite covering of $I_{0}$ by such neighbourhoods. Consequently, there is an integer $N_{0}$ such that

$$
\left|f_{n}(x)-f(x)\right|<2 \varepsilon \text { for } x \in I_{0} \text { and } n>N \text {. }
$$

Thus $\left\{f_{\boldsymbol{n}}\right\}$ converges almost uniformly to $f$

5) For the definition of asymptotic convergence, see e. g. P. H. Halmos, Measure Theory, New York 1950, p. 91.

- This notion is due to P. Montel, Legons sur les familles normales des fonctions analytiques, Paris 1927, p. 26.
2. Monotonic functions. Theorem (*) can be proved in the following stronger form:

Theorem $\boldsymbol{I}$. If a sequence $\left\{f_{n}\right\}$ of monotonic functions approximates pointwise a continuous function $f$, then $\left\{f_{n}\right\}$ converges almost uniformly to $f$.

It is sufficient to prove that every sequence $\left\{f_{n}\right\}$ of non-decreasing functions satisfies the condition (1) of Lemma 3.

Let $\varepsilon>0$ and $x_{0} \in I$. The function $f$ being continuous, there is an $\eta>0$ such that

$$
\left|f(x)-f\left(x_{0}\right)\right|<\frac{\varepsilon}{2} \text { if }\left|x-x_{0}\right|<2 \eta .
$$

Let $N$ be an integer such that, for $n>N$, the set

$$
A_{n}=\underset{x}{E}\left(\left|f_{n}(x)-f(x)\right|<\frac{\varepsilon}{2}\right)
$$

is $\frac{\eta}{2}$-dense. There are points $x_{1}, x_{2} \in A_{n}$ such that

$$
x_{0}-2 \eta<x_{1}<x_{0}-\eta \text { and } x_{0}+\eta<x_{2}<x_{0}+2 \eta \text {. }
$$

If $\left|x-x_{0}\right|<\eta$ and $n>N$, we infer from (1), (2), and (3) that

$f\left(x_{0}\right)-\varepsilon<f\left(x_{1}\right)-\frac{\varepsilon}{2}<f_{n}\left(x_{1}\right) \leqslant f_{n}(x) \leqslant f_{n}\left(x_{2}\right)<f\left(x_{2}\right)+\frac{\varepsilon}{2}<f\left(x_{0}\right)+\varepsilon$.

Hence

$$
\left|f_{n}(x)-f\left(x_{0}\right)\right|<\varepsilon \text { for } n>N \text { and }\left|x-x_{0}\right|<\eta \text {, q. e. d. }
$$

3. Convex functions. A function $f$ is said to be convex if $f(x) \leqslant \psi\left(x ; x_{1}, f\left(x_{1}\right) ; x_{2}, f\left(x_{2}\right)\right)$ for $x_{1} \leqslant x \leqslant x_{2}, x_{1}, x_{2} \in I$

where $\psi\left(x ; x_{1}, y_{1} ; x_{2}, y_{2}\right)$ is the linear function, $i . e$. which has the value $y_{i}$ at the point $x_{i}, i=1,2$.

This definition of convexity may be generalized ${ }^{7}$ ). Instead of linear functions we can take another class $\Phi$ of functions.

Let $\Phi$ be a class of functions on $I$, such that

(a) every function $\varphi \in \Phi$ is continuous;

(b) given numbers $x_{1}, x_{2}, y_{1}, y_{2}\left(x_{1}, x_{2} \in I, x_{1} \neq x_{2}\right)$, there is exactly one function $\varphi \in \Phi$ such that $\varphi\left(x_{i}\right)=y_{i}, i=1,2$.

This function $\varphi(x)$ will be denoted by $\varphi\left(x ; x_{1}, y_{1} ; x_{2}, y_{2}\right)$.

A function $f$ is said to be $\Phi_{\text {-convex }}$ if (for every $x_{1}, x_{2} \in I, x_{3}<x_{2}$ ) we have

$$
f(x) \leqslant \varphi\left(x ; x_{1}, f\left(x_{1}\right) ; x_{2}, f\left(x_{2}\right)\right) \text { for } x_{1} \leqslant x \leqslant x_{2} .
$$

7) E. F. Beckenbach, Generalized Convex Funetions, Bull. Amer. Math. Soc. 43 (1937), pp. 363-371. 
The conditions (a) and (b) imply the following properties ${ }^{8}$ ) of $\Phi$ (where $q_{1}, \varphi_{2} \in \Phi, x_{1}, x_{2} \in I, x_{1}<x_{2}$ ):

(i) If $\varphi_{1}\left(x_{1}\right)<\varphi_{2}\left(x_{1}\right)$ and $\varphi_{1}\left(x_{2}\right)<\varphi_{2}\left(x_{2}\right)$, then $p_{1}(x)<p_{2}(x)$ for $x_{1} \leqslant x \leqslant x_{2}$.

(ii) If $\varphi_{1}\left(x_{1}\right)<\varphi_{2}\left(x_{1}\right)$ and $\varphi_{1}\left(x_{2}\right)>\varphi_{2}\left(x_{2}\right)$, then $\varphi_{1}(x)<\varphi_{2}(x)$ for $a<x \leqslant x_{1}$ and $\varphi_{1}(x)>\varphi_{2}(x)$ for $x_{2} \leqslant x<b$. Analogously, if $\varphi_{1}\left(x_{1}\right)>\varphi_{2}\left(x_{1}\right)$ and $\varphi_{1}\left(x_{2}\right)<\varphi_{2}\left(x_{2}\right)$, then $\varphi_{1}(x)>\varphi_{2}(x)$ for $a<x \leqslant x_{1}$ and $\varphi_{1}(x)<\varphi_{2}(x)$ for $x_{2} \leqslant x<b$.

(iii) Given numbers $y_{1}, y_{2}$ and $\varepsilon>0$, for every closed interval $I_{0} C I$ there exists an $\eta>0$ such that

$$
\left|\varphi\left(x ; x^{\prime}, y^{\prime} ; x^{\prime \prime}, y^{\prime \prime}\right)-\varphi\left(x ; x_{1}, y_{1} ; x_{2}, y_{2}\right)\right|<\varepsilon
$$

for every $x \in I_{0},\left|x^{\prime}-x_{1}\right|<\eta,\left|x^{\prime \prime}-x_{2}\right|<\eta,\left|y^{\prime}-y_{1}\right|<\eta$ and $\left|y^{\prime \prime}-y_{2}\right|<\eta$.

The $\Phi$-convex functions have some properties similar to those of the convex function in the usual sense. For instance,

(iv) If $f$ is $\Phi$-convex and $x_{1}, x_{2} \in I, x_{1}<x_{2}$, then

$f(x) \geqslant \varphi\left(x ; x_{1}, f\left(x_{1}\right) ; x_{2}, f\left(x_{2}\right)\right)$ for $a<x \leqslant x_{1}$ and $x_{2} \leqslant x<b$.

(v) Every $\Phi$-convex function is continuous $\left.{ }^{9}\right)$.

Lemma 1. Let $F$ be a set of $\Phi$-convex functions. If $\varepsilon>0$, $x_{1}<x_{0}<x_{2}, x_{1}, x_{2} \in I$, and if, for every $f \in F$, we have

$$
\left|f\left(x_{i}\right)-y_{i}\right|<\frac{\varepsilon}{2} \quad \text { for } \quad i=0,1,2
$$

then there is an $\eta>0$ sueh that $\left|f(x)-y_{0}\right|<\varepsilon$ for every $f \in F$ and $\left|x-x_{0}\right|<\eta$. Since the functions

$$
\begin{aligned}
& \varphi_{1}(x)=\varphi\left(x ; x_{1}, y_{2}+\frac{\varepsilon}{2} ; x_{0}, y_{0}+\frac{\varepsilon}{2}\right), \\
& \varphi_{2}(x)=\varphi\left(x ; x_{0}, y_{0}+\frac{\varepsilon}{2} ; x_{2}, y_{2}+\frac{\varepsilon}{2}\right), \\
& \varphi_{3}(x)=\varphi\left(x ; x_{1}, y_{1}+\frac{\varepsilon}{2} ; x_{0}, y_{0}-\frac{\varepsilon}{2}\right), \\
& \varphi_{n}(x)=\varphi\left(x ; x_{0}, y_{0}-\frac{\varepsilon}{2} ; x_{2}, y_{2}+\frac{\varepsilon}{2}\right),
\end{aligned}
$$

8) Loc. cit. ").

-) There are other definitions of relative convexity (E. F. Beckenbach and R. H. Bing, On Generalized Convex Functions, Trans. Am. Math. Soc 58 (1945), pp. 220-230) such that the functions in $\Phi$ can be non-continuous; but then pointwise convergence does not imply almost uniform convergence. are continnous, there is an $\eta_{0}>0$ such that

(2) $\quad\left|\varphi_{p}(x)-\varphi_{p}\left(x_{0}\right)\right|<\frac{\varepsilon}{2}$ for $p=1,2,3,4$ and $\left|x-x_{0}\right|<\eta_{0}$.

Let $\eta=\min \left(\eta_{0}, x_{0}-x_{1}, x_{2}-x_{0}\right)$. It follows from the definition of the $\Phi$-convexity and from (i), (ii), (1) and (2) that, for ever $f \in \boldsymbol{F}$, the condition $x_{0}-\eta<x \leqslant x_{0}$ implies

and

$$
\begin{gathered}
f(x)-y_{0} \leqslant q\left(x ; x_{1}, f\left(x_{1}\right) ; x_{0}, f\left(x_{0}\right)\right)-y_{0}<\varphi_{1}(x)-y_{0}= \\
\left(\varphi_{1}(x)-\varphi_{1}\left(x_{0}\right)\right)+\left(\varphi_{1}\left(x_{0}\right)-y_{0}\right)<\varepsilon
\end{gathered}
$$

$$
\begin{gathered}
f(x)-y_{0} \geqslant \varphi\left(x ; x_{0}, f\left(x_{0}\right) ; x_{2}, f\left(x_{2}\right)\right)-y_{0}>\varphi_{4}(x)-y_{0}= \\
\left(\varphi_{4}(x)-\varphi_{4}\left(x_{0}\right)\right)+\left(\varphi_{4}\left(x_{0}\right)-y_{0}\right)>-\varepsilon .
\end{gathered}
$$

Analogously, if $x_{0} \leqslant x<x_{0}+\eta$, then

$$
f(x)-y_{0} \leqslant \varphi_{2}(x)-y_{0}<\varepsilon \text { and } f(x)-y_{0} \geqslant q_{3}(x)-y_{0}>-\varepsilon .
$$

Consequently, for every $f \in F$,

$$
\left|f(x)-y_{0}\right|<\varepsilon \text { if }\left|x-x_{0}\right|<\eta,
$$

Lemma 5. If $\left\{f_{n}\right\}$ is a sequence of $\Phi$-convex functions and $\lim f_{n}(x)=f(x)$ for every $x \in I$, then $f(x)$ is continuous and $\left\{f_{n}\right\}$ converges uniformly to $f$.

It is sufficient to prove that the condition (1) of Lemma 3 is satisfied.

Let $\varepsilon>0$ and $x_{0} \in I$. Since $I$ is open, there are $x_{1}, x_{2} \in I$, $x_{1}<x_{0}<x_{2}$. Let $N$ be an integer such that

$$
\left|f_{n}\left(x_{i}\right)-f\left(x_{i}\right)\right|<\frac{\varepsilon}{2} \text { for } n>N, \quad i=0,1,2 .
$$

By Lemma t there is an $\eta>0$ such that

$$
\left|f_{n}(x)-f\left(x_{0}\right)\right|<\varepsilon \text { if } n>N \text { and }\left|x-x_{0}\right|<\eta \text {, q. e. d. }
$$

Lemma 6. For every number m and for every closed interval $I_{0} \subset I$ there is a function of $\in \Phi$ such that $\varphi(x)>m$ for $x \in I_{\mathbf{0}}$.

Let $c$ and $d$ be the end points of $I_{0}, c<d$, and let

and

$$
\varphi_{n}(x)=\varphi(x ; c, n ; d, n)
$$

$$
Q_{n}=E_{x \in I_{n}}\left(\varphi_{n}(x) \geqslant m\right)
$$


It is sufficient to prove that $Q_{n_{0}}=0$ for an integer $n_{0}$. In fact, the function $\varphi_{n_{0}}(x)$ is then the required one.

Suppose the contrary, $i$. e. $Q_{n} \neq 0$ for all positive integers $n$. Since $\varphi_{n+1}(x)>\varphi_{n}(x)$ for $x \in I_{0}$ by (i), we have $Q_{n+1} \subset Q_{n}$. The sets $Q_{n}$ being compact, there is a point $x_{0} \in Q_{n}$ for $n=1,2, \ldots, i . e$.

$$
q_{n}\left(x_{0}\right) \leqslant m \text { for } n=1,2, \ldots
$$

Let $n_{0}$ be such an integer that

and

$$
\varphi_{n_{0}}(c)=n_{0}>\varphi\left(c ; x_{0}, m+1 ; l, m+1\right)
$$

$$
\tau_{n_{0}}(d)=n_{0}>\psi\left(d ; x_{0}, m+1 ; d, m+1\right) \text {. }
$$

By (i) we have

$$
\varphi_{\boldsymbol{n}_{0}}\left(x_{\mathbf{0}}\right)>\varphi\left(x_{0} ; x_{0}, m+1 ; d, m+1\right)=m+1 .
$$

which contradicts (3).

Lemma $\%$. Let $F$ be a set of $\Phi$-convex functions, let $I_{0}=\left\langle a^{\prime}, b^{\prime}\right\rangle \subset I$ be a closed interval, and let $\eta=\frac{1}{3} \min \left(a^{\prime}-a, b-b^{\prime}\right)$. If there is a number $m$ such that the sets

$$
A(f)=\underset{x}{E}(f(x)<m)
$$

are $\eta$-dense for every $f \in F$, then there is a number $M$ such that $f(x)<M$ for every $f \in F$ and for every $x \in I_{0}$.

By Lemma 6 there is a function $p_{0} \in \Phi$ such that $p_{0}(x)>m$ if $a+\eta \leqslant x \leqslant b-\eta$. Let $M$ be the least upper bound of $\varphi_{0}$ in the interval $a+\eta \leqslant x \leqslant b-\eta$.

Let $f \in F$. Since $A(f)$ is $\eta$-dense, there are points $x^{\prime}, x^{\prime \prime} \in A(f)$ such that

$$
a+\eta<a^{\prime}<a+3 \eta \leqslant a^{\prime}, \quad b^{\prime} \leqslant b-3 \eta<x^{\prime \prime}<b-\eta .
$$

The property (i) implies that

$$
f(x) \leqslant \varphi\left(x ; x^{\prime}, f\left(x^{\prime}\right) ; x^{\prime \prime}, f\left(x^{\prime \prime}\right)\right)<\varphi\left(x ; x^{\prime}, m ; x^{\prime \prime}, m\right) \leqslant \varphi_{0}(x) \leqslant M \Gamma
$$

for every $x \in I_{0}$.

Theorem II. If a sequence $\left\{f_{n}\right\}$ of $\Phi$-convex functions approximates pointwise a continuous function $f$, then $\left\{f_{n}\right\}$ converges almost
uniformly to $f$.

By Lemma $\check{5}$ it is sufficient to prove that $\lim f_{n}\left(x_{0}\right)=f\left(x_{0}\right)$
every $x_{0} \in I$.
Let $\varepsilon>0$, and let $I_{0}=\left\langle a^{\prime}, b^{\prime}\right\rangle$ be a closed interval, $a<a^{\prime}<x_{0}<b^{\prime}<b$. Let $\eta=\frac{1}{3} \min \left(a^{\prime}-a, b-b^{\prime}\right)$. There is an integer $N_{1}$ such that the set

$$
\underset{x}{E}\left(\left|f_{n}(x)-f(x)\right|<1\right)
$$

is $\frac{\eta}{2}$-dense for $n>N_{1}$. Since $f$ is continuous, there is a number $m$ such that $f(x) \leqslant m-1$ for $a+\frac{\eta}{2} \leqslant x \leqslant b-\frac{\eta}{2}$. Hence the set

$$
E_{\boldsymbol{x}}\left(f_{n}(x) \leqslant m\right)
$$

is $\eta$-dense for $n>N_{1}$. By Lemma 7 there is a number $M$ such that $f_{n}(x) \leqslant M$ for $x \in I_{0}$; and $n>N_{1}$. Let

$$
\begin{aligned}
& \varphi_{1}(x)=\varphi\left(x ; x_{0}, f\left(x_{0}\right) ; b^{\prime}, M\right) \\
& \varphi_{2}(x)=\varphi\left(x ; a^{\prime}, M ; x_{0}, f\left(x_{0}\right)\right) .
\end{aligned}
$$

By (iii) there is a $\delta>0\left(x_{0} \pm \delta \epsilon I_{0}\right)$ such that

$$
\text { (4) } \quad\left|\varphi_{1}(x)-\varphi\left(x ; x_{1}, f\left(x_{0}\right)+\vartheta ; b^{\prime}, M\right)\right|<\varepsilon
$$

$$
\left|\varphi_{2}(x)-p\left(x ; a^{\prime}, M ; x_{1}, f\left(x_{0}\right)+\vartheta\right)\right|<\varepsilon
$$

if $\left|x_{1}-x_{0}\right|<\delta,|\vartheta|<\delta$ and $a^{\prime} \leqslant x \leqslant b^{\prime}$.

Since $f$ is continuous, there is a $\gamma>0$ such that

$$
\left|f(x)-f\left(x_{0}\right)\right|<\frac{\delta}{2} \text { if }\left|x-x_{0}\right|<\gamma .
$$

Let $\mu=\min (\delta, \gamma)$. There is an integer $N\left(>N_{1}\right)$ such that the sets

$$
A_{n}=\underset{x}{E}\left(\left|f_{n}(x)-f(x)\right|<\frac{\delta}{2}\right)
$$

are $\frac{\mu}{2}$-dense for $n>N$. Consequently, if $n>N$, there is a point $x_{n} \in A_{n}$ such that $x_{0}-\mu<x_{n}<x_{0}$. We have from (i), (4), (6), (7)

$$
\begin{gathered}
f_{n}\left(x_{0}\right) \leqslant \varphi\left(x_{0} ; x_{n}, f_{n}\left(x_{n}\right) ; b^{\prime}, M\right)<\varphi\left(x_{0} ; x_{n}, f\left(x_{n}\right)+\frac{\delta}{2} ; b^{\prime}, M\right) \\
<\varphi\left(x_{0} ; x_{n}, f\left(x_{0}\right)+\delta ; b^{\prime}, M\right)<\varphi_{1}\left(x_{0}\right)+\varepsilon=f\left(x_{0}\right)+\varepsilon .
\end{gathered}
$$


It follows from (i), (ii), (5), (6), (7) that

$$
\begin{gathered}
f_{n}\left(x_{0}\right) \geqslant \varphi\left(x_{0} ; a^{\prime}, M ; x_{n}, f_{n}\left(x_{n}\right)\right)>\varphi\left(x_{0} ; a^{\prime}, M ; x_{n}, f\left(x_{n}\right)-\frac{\delta}{2}\right) \\
>p\left(x_{0}^{\prime} ; a^{\prime}, M ; x_{n}, f\left(x_{0}\right)-\delta\right)>p_{2}\left(x_{0}\right)-\varepsilon=f\left(x_{0}\right)-\varepsilon .
\end{gathered}
$$

Hence $\left|f_{n}\left(x_{0}\right)-f\left(x_{0}\right)\right|<\varepsilon$ for $n>N$, which proves that $\lim f_{n}\left(x_{0}\right)=f\left(x_{0}\right)$, q. e. $d$.

If we replace the pointwise approximation in Theorem II by convergence on a dense subset or by asymptotic convergence, we can omit the assumption that $f$ is continuous. This is the subject of Theorems III and IV below. To prove those theorems we shall first establish the two following lemmas:

Lemma 8 . Let $F$ be a set of $\Phi$-convex functions and let $F(x)$ $b e$ the set of all numbers $f(x)$ where $f \in F(x \in I)$. If all the sets $F(x)$ are simultaneously bounded from above by a number $M$, and if, for an $x_{0} \in I$, the set $F\left(x_{0}\right)$ is bounded from below by a number $m$, then every set $F(x)(x \in I)$ is bounded from below.

Let $x \in I$. If $x<x_{0}$, let $x_{1} \in I, x_{1}>x_{0}$. By (ii) and (iv)

$$
f(x) \geqslant \varphi\left(x ; x_{0}, f\left(x_{0}\right) ; x_{1}, f\left(x_{1}\right)\right) \geqslant q\left(x ; x_{0}, m ; x_{1}, M\right) .
$$

If $x>x_{0}$, let $x_{2} \in I, x_{0}>x_{2}$. By (ii) and (iv)

$$
f(x) \geqslant \varphi\left(x ; x_{2}, f\left(x_{2}\right) ; x_{0}, f\left(x_{0}\right)\right) \geqslant \varphi\left(x ; x_{2}, M ; x_{0}, m\right) .
$$

The Lemma is established.

Lemma 9. Let $\left\{f_{n}\right\}$ be a sequence of $\Phi$-convex functions such that $f(x)=\varlimsup \lim f_{n}(x)$ is finite for every $x \in I$. Then $f(x)$ is $\Phi$-convex. Let $x_{1}<x<x_{2}, x_{1}, x_{2} \in I$. Given $\varepsilon>0$, there is an integer $N$ such that $f_{n}\left(x_{i}\right)<f\left(x_{i}\right)+\varepsilon$ for $n>N, i=1,2$. We have by (i)

$$
\begin{aligned}
& f_{n}(x) \leqslant \varphi\left(x ; x_{1}, f_{n}\left(x_{1}\right) ; x_{2}, f_{n}\left(x_{2}\right)\right)<\varphi\left(x ; x_{1}, f\left(x_{1}\right)+\varepsilon ; x_{2}, f\left(x_{2}\right)+\varepsilon\right) . \\
& \text { Consequently }
\end{aligned}
$$

$$
f(x) \leqslant \varphi\left(x ; x_{1}, f\left(x_{1}\right)+\varepsilon ; x_{2}, f\left(x_{2}\right)+\varepsilon\right) .
$$

The proposition (iii) means that $\varphi\left(x ; x_{1}, y_{1} ; x_{2}, y_{2}\right)$ is a continuous function with respect to all the variables $x_{1}, y_{1}, x_{2}, y_{2}$ simultaneously. Hence, when $\varepsilon \rightarrow 0$, we obtain

$$
f(x) \leqslant \varphi\left(x ; x_{1}, f\left(x_{1}\right) ; x_{2}, f\left(x_{2}\right)\right),
$$

Theorem III. Let $\left\{f_{n}\right\}$ be a sequence of $\Phi$-convex functions. If $\lim f_{n}(x)$ exists for $x$ belonging to a dense subset SCI, then the sequence $\left\{f_{n}\right\}$ converges almost uniformly to a function $f$.

Let $I_{0}=\left\langle a^{\prime}, b^{\prime}\right\rangle \subset I$ be a closed interval, and let

$$
\eta=\frac{1}{3} \min \left(a^{\prime}-a, b-b^{\prime}\right) \text {. }
$$

Let $A$ be a finite $\eta$-dense subset of $S$, and let

$$
m=\max _{x \in A}\left(\lim f_{n}(x)\right)+1 \text {. }
$$

There is an integer $N$ such that $\left|\lim f_{n}(x)-f_{n}(x)\right|<1$ for $n>N$ and $x \in A$. Consequently, the sets

$$
E_{x}\left(f_{n}(x)<m\right) \text { where } n>N
$$

are $\eta$-dense since they contain the $\eta$-dense set $A$. By Liemma 7 all functions $f_{n}(n>N)$ are simultaneously bounded from above in $I_{0}$.

The interval $I_{0}$ being arbitrary, we infer that the set $F(x)$ of all numbers $f_{n}(x)(n=1,2, \ldots)$ is bounded from above for every $x \in I$. Since $F(x)$ is bounded from below for each $x \in S$, we infer from Lemma 8 that $F(x)$ is bounded from below for every $x \in I$. Consequently $f(x)=\varlimsup \lim f_{n}(x)$ is finite, thus, by Lemma 9 , the function $f$ is $\Phi$-convex and continuous (see (v)). By Lemma 1 , the sequence $\left\{f_{n}\right\}$ approximates pointwise the function $f$. By Theorem II, the sequence $\left\{f_{n}\right\}$ converges almost uniformly to $f$.

Theorem IV. If a sequence $\left\{f_{n}\right\}$ of $\Phi$-convex functions converges asymptotically, then it converges almost uniformly to a continuous function $f$.

The sequence $\left\{f_{n}\right\}$ contains a subsequence $\left\{f_{m_{n}}\right\}$ which converges almost everywhere. By Theorem III, the sequence $\left\{f_{m_{n}}\right\}$ converges almost uniformly to a continuous function $f$. Clearly $f=\lim$ as $f_{n}$. Consequently $\left\{f_{n}\right\}$ approximates $f$ pointwise, by Lemma 2 . By Theorem II the sequence $\left\{f_{n}\right\}$ converges almost uniformly to $f$.

4. Functions with non-negative $p$-th difference. The first difference of a function $f$ is

$$
\Delta^{1}(x, h, f)=\Delta(x, h, f)=f(x+h)-f(x) .
$$

By induction, the $p$-th difference of $f$ is

$$
\Delta^{p}(x, h, f)=\Delta^{p-1}(x+h, h, f)-\Delta^{p-1}(x, h, f) .
$$


Clearly

$$
\begin{aligned}
& \Delta^{p}(x, h, f)=\sum_{k=0}^{p}(-1)^{p-k}\left(\begin{array}{l}
p \\
k
\end{array}\right) f(x+k h), \\
& \Delta^{p}(x, 2 h, f)=\sum_{k=0}^{p}\left(\begin{array}{l}
1 \\
k
\end{array}\right) \Delta^{p}(x+k h, h, f),
\end{aligned}
$$

$$
\Delta^{p}(x, h, f+g)=\Delta^{p}(x, h, f)+\Delta^{p}(x, h, g) .
$$

The $p$-th difference $\Delta^{p}(x, h, f)$ is defined only for such $x \in I$ that $x+p h \in I$.

We shall consider only the case $h>0$. Since $\Delta^{p}(x, h, f)=$ $=(-1)^{p} \Delta^{p}(x+h p,-h, f)$, the above restriction is unessential.

In the sequel $h$ is always a positive number.

Let $\Psi_{p}$ be the class of all functions on $I$ with non-negative $p$-th difference, $i$. e. the class of all $f$ such that $\Delta^{p}(x, h, f) \geqslant 0$ for arbitrary $x \in I$ and $h>0$ for which the $p$-th difference is defined. Evidently $\Psi_{1}$ is the class of all non-decreasing functions, and $\Psi_{2}$ is the class of all convex functions.

Lemma 10. Let $f \in \Psi_{p+1}$ and let $x_{0} \in I$ and $h_{0}>0$ be such that $\Delta^{p}\left(x_{0}, h_{0}, f\right)>0$. Then

$$
\Delta^{p}\left(x_{0}+p h_{0}, \frac{h_{0}}{2^{m}}, f\right)>0 \quad \text { for } \quad m=0,1,2, \ldots
$$

Since $f \in \Psi_{p+1}$, we have

(2) $\quad \Delta^{p}(x+k h, h, f) \geqslant \Delta^{p}(x+l h, h, f)$ if $k \geqslant l, \quad k, l=0,1,2, \ldots$

By (ii),

$$
\sum_{k=0}^{p}\left(\frac{p}{k}\right) \Delta^{p}\left(x_{0}+k \frac{h_{0}}{2}, \frac{h_{0}}{2}, f\right)=\Delta^{p}\left(x_{0}, h_{0}, f\right)>0
$$

Hence, by (2), $\Delta^{p}\left(x_{0}+p \frac{h_{0}}{2}, \frac{h_{0}}{2}, f\right)>0$. Analogously, we obtain by induction on $m$ that

$$
\Delta^{p}\left(x_{0}+p\left(\frac{h_{0}}{2}+\ldots+\frac{h_{0}}{2^{m}}\right), \frac{h_{0}}{2^{m}}, f\right)>0 .
$$

The last inequality and (2) directly imply (1).
Lemma 11. Let $F \subset \Psi_{p+1}$, let $I_{0}=\left(a^{\prime}, b^{\prime}\right)$ be an open subinterval of $I$, and let $|f(x)|<M$ for every $x \in I_{0}$ and for every $f \in F$. Given two numbers $x_{1}, x_{2} \in I_{0}\left(x_{1}<x_{2}\right)$, there is a continuous function $g$ on $I$ such that, for every $f \in F$,

$$
\begin{array}{lll}
\Delta^{p}(x, h, f+g)>0 & \text { if } & x_{1}<x<b, \\
\Delta^{p}(x, h, g-f)>0 & \text { if } & a<x<x_{2} .
\end{array}
$$

It is sufficient to put

$$
g(x)=\frac{2^{p} M}{p ! d^{p}} x^{p}, \text { where } \quad d=\frac{1}{2 p} \min \left(x_{1}-a^{\prime}, b^{\prime}-x_{2}\right) .
$$

Clearly

$$
\begin{gathered}
\Delta^{p}(x, h, g)=2^{p} M^{p}\left(\frac{h}{d}\right)^{p} \\
\Delta^{p+1}(x, h, g)=0
\end{gathered}
$$

Proof of (3). Consider first the case where $d \leqslant h<2 d$. Let $0 \leqslant u \leqslant d$. We have

$$
\Delta^{p}\left(a^{\prime}+d+u, h, f\right)>-2^{p} M
$$

on account of (i) since $a^{\prime}+d+u+k h \in I_{0}$ for $k=0,1,2, \ldots, p$.

Consequently, by (iii) and (5),

$$
\Delta^{p}\left(a^{\prime}+d+u, h, f+g\right)>-2^{p} M+2^{p} M\left(\frac{h}{d}\right)^{p} \geqslant 0 .
$$

Since $f \in \Psi_{p+1}$ and $(6)$, we infer that $f+g \epsilon \Psi_{p+1}$. Hence, by Lemma 10 and (7),

$$
\Delta^{p}\left(a^{\prime}+d+u+p h+\frac{k h}{2^{m}}, \frac{h}{2^{m}}, f+g\right) \geqslant \Delta^{p}\left(a^{\prime}+d+u+p h, \frac{h}{2^{m}}, f+g\right)>0
$$

for $m=0,1,2, \ldots, k=0,1,2, \ldots, 0 \leqslant u \leqslant d \leqslant h<2 d$.

Since every number $x>x_{1}$ can be represented in the form

$$
x=a^{\prime}+d+u+p h+k \frac{h}{2^{m}},
$$

where $0 \leqslant u \leqslant d$ and $k$ is a positive integer, we obtain

$$
\Delta^{p}\left(x, \frac{h}{2^{m}}, f+g\right)>0 \quad \text { if } \quad x_{1}<x \in I, \quad d \leqslant h<2 d \quad \text { and } \quad m=0,1,2, \ldots
$$

Fundamenta Mathematicae. T. XXXIX. 
Since every number from the open interval $(0,2 d)$ may be represented in the form $h / 2^{m}$, where $d \leqslant h<2 d$ and $m$ is one of the numbers $0,1,2, \ldots$, we infer that

$$
\Delta^{p}(x, \dot{h}, f+g)>0 \quad \text { if } \quad x_{1}<x \in I \text { and } 0<h<2 d .
$$

The formula (ii) implies that the restriction $h<2 d$ can be omitted. The inequality (3) is proved.

Proof of (4) is analogous to that of (3).

Lemma 11 is very useful in inductive proofs of theorems which generalizo the properties of convex functions to the cases of functions in $\Psi_{p}(p \geqslant 2)$. $T$. $g$. the following theorem is a simple consequence of Lemma $1 \mathrm{l}$ :

Theorem $\left.V^{10}\right)$. Every function $f \in \Psi_{p}(p \geqslant 2)$ bounded on an open interval $I_{0} C I$ is continuous in $I$.

The proof is by induction on $p$. If $p=2, f$ is convex and, consequently, continuous ${ }^{11}$ ).

Suppose Theorem $V$ is true for $p$. Let $f \epsilon \Psi_{p+1}$ be bounded on an open interval $I_{0} \subset I$. Let $x_{1}, x_{2} \in I_{0}, x_{1}<x_{2}$. By Lemma 11 there is a function $g$ continuous in $I$ such that

and

$$
\begin{array}{lll}
\Delta^{p}(x, h, f+g)>0 & \text { if } & x>x_{1}, \\
\Delta^{p}(x, h, g-f)>0 & \text { if } & x<x_{2} .
\end{array}
$$

By the inductive hypothesis, $f+g$ is continuous on the interval $\left(x_{1}, b\right)$, and $g-f$ is continuous in $\left(a, x_{2}\right)$. Consequently, $f$ is continuous in $I$, q. e. $d$.

Theorem VI. Suppose $\left\{f_{n}\right\}$ is a sequence of functions in $\Psi_{p}(p \geqslant 2)$, and $\left|f_{n}(x)\right|<M$ for $x$ in an open interval $I_{0} \subset I, n=1,2, \ldots$

If one of the following conditions is satisfied:

(a) $\left\{f_{n}\right\}$ approximates pointwise a continuous function $f$;

(b) $\lim f_{n}(x)$ exists for $x$ in a dense subset of $I$;

(c) $\left\{f_{n}\right\}$ converges asymptotically in $I$;

then $\left\{f_{n}\right\}$ converges almost uniformly in $I$ to a continuous function $(=f$ in the case (a)).

The proof is by induction on $p$.

If $p=2$, the functions $f_{n}$ are convex. Theorem $V$ is then a particular case of Theorems II, III, IV.

$\left.{ }^{10}\right)$ Proved by T. Popoviciu (Sur quelques propriétés des fonctions d'une ou deux variables réelles, Mathematica 8 (1934), pp. 1-85, in particular p. 56 under the stronger assumption that $f$ is bounded in the whole interval $I$.

11) F. Bernstein and G. Doetsch, Zur Theorie der konvexen Funktionen, Math. Annalen 76 (1915), pp. 514-526.
Suppose Theorem $\mathrm{V}$ is trie for an integer $p \geqslant 2$. We shall prove that it is true for $p+1$.

Let $\left\{f_{n}\right\}$ be a sequence of functions in $\Psi_{p+1}$ both bounded in an open interval $I_{0} \subset I$, and let $x_{1}, x_{2} \in I_{0}, x_{1}<x_{2}$. By Lemma 11 there is a continuous function $g$ such that

and

$$
\begin{aligned}
& \Delta^{p}\left(x, h, f_{n}+g\right)>0 \quad \text { if } \quad x>x_{1} \\
& \Delta^{p}\left(x, h, g-f_{n}\right)>0 \quad \text { if } \quad x<x_{2} .
\end{aligned}
$$

By the induction hypothesis, the sequence $\left\{f_{n}+g\right\}$ converges almost uniformly in the interval $\left(x_{1}, b\right)$, and the sequence $\left\{g-f_{n}\right\}$ converges almost uniformly in $\left(a, x_{2}\right)$. Consequently $\left\{f_{n}\right\}$ converges almost uniformly in $I=(a, b)$, q. e. d

5. Subharmonic functions. The following examples show that, in the case of subharmonic functions ${ }^{12}$ ) which can be considered as generalization of a convex function to the case of functions of two variables, pointwise approximation does not imply convergence, and weaker kinds of convergence do not imply stronger kinds.

1. The sequence of subharmonic functions

$$
u_{n}(x, y)=\frac{1}{n} \log \left[x+\frac{1}{n}+y\right]
$$

converges everywhere to 0 , but not almost uniformly in a neighbourhood of $(0,0)$.

2. The sequence of subharmonic functions

$$
u_{n}(x, y)=\max \left[c_{n}, \frac{1}{n} \log (x+y)\right]
$$

where $\left\{c_{n}\right\}$ is a divergent sequence of negative numbers, converges if $(x, y) \neq(0,0)$ and diverges at the point $(0,0)$.

3. Let $K$ be a fixed square. Divide $K$ into four squares $K_{1}, K_{2}, K_{3}, K_{4}$; divide each of these four squares into four squares denoted by $K_{5}, K_{6}, \ldots, K_{20} ;$ and so on to infinity. We obtain an in-

12) For the definition of subharmonic functions, see T. Radó, Subharmonio Functions, Berlin 1937. 
finite sequence of squares $K_{n}$ with diameters $2 h_{n}$ and with middle points $\left(x_{n}, y_{n}\right)$. The sequence of subharmonic functions

$$
u_{n}(x, y)=\frac{1}{\log h_{n}^{2}} \log \left[x-x_{n}+y-y_{n}\right]
$$

converges asymptotically to 0 in $K$, but it diverges at every point in $K$.

4. Divide a fixed square $K$ into four squares. Denote by $K_{1,1}$ and $K_{1,2}$ the squares lying on the principal diagonal of $K$, and by $K_{1,3}$ and $K_{1,4}$ the two remaining squares. Now divide each of the 4 squares $K_{1, j}(j=1, \ldots, 4)$ into four squares and denote the squares thus obtained by $K_{2, i}\left(i=1, \ldots, 4^{2}\right)$ so that the squares $K_{2, t}\left(i=1, \ldots, \frac{1}{2} \cdot 4^{2}\right)$ lie on principal diagonals of $K_{1, j}$. Continue this process to infinity so that $K_{n+1, i}\left(i=1, \ldots, \frac{1}{2} \cdot 4^{n+1}\right)$ lie on principal diagonals of $K_{n, j}$ $\left(j=1, \ldots, 4^{n}\right)$. Let $2 h_{n}$ be the diameter of $K_{n, j}$, and let $\left(x_{n, j}, y_{n, j}\right)$, be the middle point of $K_{n, 1}$.

The sequence of subharmonic functions

$$
u_{n}(x, y)=\sum_{p=1}^{\frac{1}{2} \cdot 4^{n}} \frac{1}{\log h_{n}^{2}} \log \left(x-x_{n, p}+y-y_{n, p}\right)
$$

approximates pointwise the function $u(x, y)=1$ in $K$, but it does not converge asymptotically to $u(x, y)$.

\section{On Partition of an Ordered Continuum.}

By

\author{
Jos ef Novák (Praha).
}

The result of the consecutive division of an ordered continuum is a system of intervals which satisfy certain conditions (Theorem 3). By these conditions - as in axioms $1^{0}-4^{0}-$ a system $\mathfrak{P}$ of intervals of an ordered continuum $C$-called a partition of $C-$ is defined. From a thorough study of these axioms a row of properties emerges concerning the partition $\mathfrak{P}$ and the ordered continuum $C$. For instance: For a given ordered continuum $C$ all the partitions have the same cardinality equal to the least cardinal $\mathrm{m}(C)$ of the set which is dense in $C$. Therefore the cardinality of the partition of $O$ is topologically invariant.

In this paper the following theorem is proved: Let $O$ be an ordered continuum. Then there exists an ordinal $\vartheta$ of power $\leqslant \mathfrak{m}(C)$ such that $C$ is similar to a lexicographically ordered set whose elements are transfinite sequences of zeros and ones of order-types $\leqslant \vartheta$ (Theorem 4). It is interesting to compare this result with the following theorem of Sierpiński ${ }^{1}$ ): For a given ordinal $v$ every ordered set of power $\kappa_{v}$ is similar to a lexicographically ordered set whose elements are transfinite sequences of zeros and ones of type $\omega_{\boldsymbol{v}}$.

We shall prove at the end of the paper that a necessary and sufficient condition for all ordered continua of power $\boldsymbol{\aleph}_{\sigma}$ to contain a point with character $c_{00}$ is the inequality: $s_{\sigma}<2^{k_{2}}$ (Theorem 5). From this it follows that every ordered continuum of power $2^{*_{0}}$ and of $\mathrm{m}(C)=\aleph_{1}$ contains a subset which is dense in $C$ and whose points have character $c_{00}$ (Corollary).

1) See W. Sierpiński, Sur une propriété des ensembles ordonnés, Fund. Math. 36 (1949), p. 56. 